\title{
Perfil audiológico de pacientes com diabetes mellitus tipo II
}

\section{Audiologic profile of patients with diabetes mellitus type II}

\author{
Juliana Mota Ferreira ${ }^{1}$, Fernanda Mônica de Oliveira Sampaio ${ }^{2}$, Joyce Monte Silva Coelho ${ }^{3}$, \\ Nádia Maria Girão Saraiva de Almeida ${ }^{4}$
}

\begin{abstract}
RESUMO
Objetivo: Pacientes acometidos por Diabetes Mellitus (DM) do tipo II constituem uma população de risco para alterações auditivas, uma vez que alterações metabólicas podem afetar tecidos nervosos e vasculares, que atingem diretamente os órgãos responsáveis pela audição. O presente estudo buscou investigar o perfil audiológico de pacientes com DM do tipo II. Métodos: Foi realizado um estudo descritivo, no qual foram avaliados 44 sujeitos (88 orelhas). A população selecionada encontrava-se na faixa etária de 25 a 65 anos de idade. Todos os sujeitos realizaram avaliação audiológica, sendo submetidos a audiometria tonal liminar, audiometria vocal e imitância acústica. Resultados: A prevalência de pacientes com DM do tipo II foi maior no sexo feminino (77,3\%). Trinta e um $(70,4 \%)$ pacientes apresentavam DM tipo do II a um período inferior a 10 anos. Trinta e três orelhas (37,5\%) apresentaram perda auditiva neurossensorial de grau leve ou moderado, com predomínio em freqüências altas. Na imitância acústica, 71 orelhas (80,7\%) apresentaram curva do tipo A, e 83 orelhas (94,3\%) tiveram presença de reflexo estapediano. Conclusão: Observou-se a ocorrência de perda auditiva em pacientes portadores de DM do tipo II, conseqüentemente, a avaliação audiológica desses pacientes é importante para o acompanhamento das alterações auditivas.
\end{abstract}

Descritores: Diabetes mellitus tipo 2/diagnóstico; Diabetes mellitus tipo 2/complicações; Glicemia; Perda auditiva/etiologia

\section{INTRODUÇÃO}

O diabetes mellitus (DM) é uma alteração metabólica crônica caracterizada por hiperglicemia e alterações no metabolismo da gordura e proteína ${ }^{(1)}$. É diagnosticada quando o corpo é incapaz de controlar efetivamente o processamento de açúcar (glicose) na corrente sanguínea, devido à deficiência de ação absoluta ou relativa da insulina ${ }^{(2)}$. Na sua manifestação clínica completa, é caracterizada por alterações metabólicas, complicações vasculares e neuropáticas ${ }^{(3)}$.

A prevalência de diabetes vem crescendo nos últimos anos, o que está associado às mudanças no estilo de vida da população. Entretanto, muitos indivíduos desconhecem ser portadores de diabetes, convivendo anos com hiperglicemia, aumentando o risco de complicações vasculares, renais, car-

Trabalho realizado na Universidade de Fortaleza - UNIFOR e no Núcleo de Atenção Médica Integrada - NAMI - Fortaleza (CE), Brasil.

(1) Pós-graduanda em Audiologia pela Universidade Federal do Ceará - UFC

- Fortaleza (CE), Brasil.

(2) Mestre, Professora do Curso de Fonoaudiologia da Universidade de Fortaleza - UNIFOR - Fortaleza (CE), Brasil.

(3) Mestre, Professora do Curso de Fonoaudiologia da Universidade de Fortaleza -UNIFOR - Fortaleza (CE), Brasil.

(4) Doutora, Docente da Escola de Saúde Pública do Ceará - ESP - Fortaleza (CE), Brasil.

Endereço para correspondência: Juliana Mota Ferreira. R. Joaquim Albano,

76, Padre Andrade, Fortaleza - CE, CEP 60356-340. E-mail: julianamf04@yahoo.com.br

Recebido em: 9/3/2007; Aceito em: 12/11/2007 díacas, neurológicas, oftalmológicas e infecciosas ${ }^{(4)}$.

No Brasil, estima-se que existam cinco milhões de indivíduos diabéticos, dos quais metade desconhece o diagnósti$\mathrm{co}^{(3,5)}$. Um levantamento realizado na cidade de Fortaleza, pela Associação Nacional de Diabéticos, revelou prevalência do diabetes mellitus na população de 30 a 69 anos de $6,48 \%{ }^{(6)}$.

Dos pacientes diabéticos, 5 a $10 \%$ não produzem insulina, condição classificada como DM do tipo I. A maioria das pessoas com o distúrbio tem DM do tipo II, caracterizado por um estado de resistência periférica à ação da insulina associada à secreção de insulina deficiente. Esses indivíduos ainda apresentam alguma insulina endógena, mas os seus níveis são insuficientes para manter a normoglicemia em função da resistência à sua ação ${ }^{(2,7)}$.

Considerado importante problema de saúde pública, o DM do tipo II ou simplesmente diabetes tipo II, é responsável por aproximadamente $90 \%$ de todos os casos e as complicações dele decorrentes comprometem a produtividade, qualidade de vida e sobrevida dos indivíduos ${ }^{(8)}$. Pode ocorrer em qualquer faixa etária, porém é mais freqüente em mulheres, com idade acima dos 40 anos, devido a maior prevalência de obesidade neste $\operatorname{sexo}^{(6)}$.

O diabetes possui evolução crônica e, dependendo do controle metabólico obtido, pode gerar complicações agudas e crônicas, que podem ser fatais. O diabetes é considerado um dos mais importantes fatores de risco para complicações macro e microvasculares, que já começam a se desenvolver de forma gradual pelos efeitos crônicos da 
hiperglicemia, ocasionando disfunção, dano e falência de vários órgãos, especialmente os olhos, rins, nervos e sistema cardiovascular $^{(4,8)}$.

Os tecidos vasculares e nervosos têm papel predominante na função auditiva; qualquer doença que tenha capacidade de causar prejuízo as suas células tem potencial para afetar negativamente os vários órgãos auditivos. A ligação entre audição e diabetes parece provável, de fato, se o suprimento sangüíneo para a cóclea e/ou centros nervosos no trajeto auditivo, incluindo o cérebro, estão afetados ${ }^{(2)}$.

Em 1975, as estimativas de prevalência de perda auditiva na população diabética variavam de 9 a $47 \%{ }^{(9)}$. Estudo recente revelou que, em relação à incidência de perda auditiva em pacientes com DM, também não há consenso na literatura, variando de zero a $93 \%{ }^{(3)}$. São essas discrepâncias que têm conduzido o desenvolvimento de várias pesquisas, na intenção de elucidar a associação referida e suas causas ${ }^{(9)}$.

A relação entre diabetes e limiares auditivos merece especial atenção. Estudos têm tentado mostrar o mecanismo fundamental pelo qual os níveis de insulina e glicose poderiam acarretar alterações da percepção auditiva ${ }^{(10)}$. Várias hipóteses têm sido sugeridas, entre elas: comprometimento da micro-circulação, fatores neuropáticos e o efeito da hiperglicemia ${ }^{(11)}$.

Neuropatia e angiopatia são afecções comuns no DM. Os fatores que podem ser causa da neuropatia são os distúrbios no metabolismo de glicose, lipídeos e vitaminas. A angiopatia pode ocorrer tanto de maneira direta, interferindo com o suprimento para a cóclea pela redução do transporte através das paredes espessadas dos capilares, como indiretamente, pela redução no fluxo de uma estreita vasculatura ou, ainda, por causar degeneração secundária do oitavo nervo craniano ${ }^{(3)}$.

Outro fator importante no estudo da relação entre diabetes e perda auditiva seria a presença de hipertensão arterial. Sabendo-se que o diabetes está entre os fatores que predispõem ao descontrole da pressão - quem tem diabetes tem duas vezes mais chances de desenvolver a hipertensão - informações sobre a relação entre ambas são de grande utilidade. A hipertensão está relacionada diretamente a um maior grau de resistência à insulina, e alguns medicamentos usados para o tratamento da hipertensão pioram essa resistência, favorecendo o aparecimento do diabetes ${ }^{(12)}$.

Algumas pesquisas, analisando pacientes com hipertensão arterial em associação com diabetes, não encontraram relação entre a presença destas patologias e a piora dos limiares audiométricos ${ }^{(13)}$, contrariando outros autores ${ }^{(14-15)}$.

Estudos da função auditiva em pacientes diabéticos mostraram que os mesmos apresentaram perda auditiva neurossensorial bilateral progressiva, com predomínio em freqüências altas ${ }^{(1,10,16)}$.

Uma pesquisa investigou a audição de 694 sujeitos com idade entre 25 e 85 anos. Nos voluntários mais velhos, a proporção de perda auditiva foi semelhante entre os diabéticos e não-diabéticos. No entanto, quando foram divididos por grupos de idades, os autores verificaram que os diabéticos abaixo de 60 anos tinham significativamente mais perda auditiva $^{(17-18)}$.

A freqüência da perda auditiva neurossensorial aumenta com a duração do diabetes, porém esta informação deve ser interpretada com cautela, já que poderia relacionar-se com a presbiacusia, própria da idade mais avançada, e não com o diabetes, embora outros estudos constatem também uma porcentagem importante de pessoas com idades inferiores a 60 $\operatorname{anos}^{(11)}$. Pesquisas relatam que a perda auditiva encontrada no diabetes não segue um padrão similar ao da presbiacusia, em função da distribuição das frequiências, sendo mais linear ${ }^{(2,9)}$.

Estudos desenvolvidos em 1997 visaram investigar a via central e periférica, em pacientes com diabetes do tipo II. Os autores concluíram que o receptor coclear é o principal afetado nos pacientes diabéticos tipo II, e que não há comprometimento das vias auditivas centrais ${ }^{(9)}$.

A experiência clínica, em muitos trabalhos, mostra relação direta entre perda de audição e diabetes ${ }^{(9,11)}$. Porém, há outros trabalhos com grande número de indivíduos e bem estruturados, que negam a existência de tal associação ou referem uma fraca associação $\mathrm{O}^{(1,3,19)}$.

Atualmente, especialistas recomendam que pessoas com diabetes realizem exames regulares para monitorar alterações na visão, função renal, sensibilidade térmica e tátil e saúde cardiovascular. A continuidade das pesquisas para definir a natureza da conexão entre perda auditiva e diabetes pode mostrar que testes específicos para perda auditiva poderiam ser incluídos como parte desses cuidados de rotina ${ }^{(2)}$.

Levando-se em consideração que o diabetes pode afetar os tecidos vasculares e nervosos, fundamentais na função auditiva adequada, surgiu o interesse de se realizar um estudo que tem como objetivo identificar o perfil audiológico em pacientes com diabetes do tipo II acompanhados no Setor de Diabetes do Núcleo de Atenção Médica Integrada (NAMI), vinculado à Universidade de Fortaleza - UNIFOR. A meta é identificar as principais queixas auditivas e alguns fatores relacionados, como tempo de evolução da doença e presença de hipertensão arterial, além de caracterizar a possível perda auditiva quanto ao tipo e ao grau. O presente estudo irá contribuir para conscientizar profissionais da saúde sobre a necessidade de se averiguar a audição desses pacientes, destacando que, quanto mais cedo as alterações auditivas forem detectadas, maiores as chances de reabilitação auditiva do indivíduo.

\section{MÉTODOS}

Trata-se de um estudo descritivo, do tipo série de casos, realizado no período de agosto de 2004 a novembro de 2005 e aprovado pelo Comitê de Ética em Pesquisa da instituição com o parecer $n^{\circ} 380 / 2005$, segundo Resolução n 196/96 (CNS).

A população do estudo foi constituída por pacientes portadores de diabetes mellitus que realizavam tratamento no Setor de Diabetes da instituição, totalizando 77 pacientes selecionados inicialmente.

Aplicou-se um formulário pré-estabelecido (anamnese), contendo as seguintes variáveis: sexo, faixa etária, queixa auditiva, tempo de evolução do diabetes, presença de hipertensão arterial, antecedentes patológicos e otológicos e história familiar de deficiência auditiva. 
Foram excluídos os pacientes com idade acima de 65 anos, buscando diminuir a interferência da idade na ocorrência de alterações auditivas. Também foram excluídos pacientes que se recusaram a participar da pesquisa, que referiram perda auditiva anterior ao diagnóstico do diabetes ou que possuíam outros fatores de risco para perda auditiva, tais como: exposição a ruído e/ou produtos tóxicos, drogas ototóxicas, lesões cranianas severas, história familiar de perda auditiva, anormalidades de orelha média, infecções otológicas, caxumba, meningite, doenças autoimunes, quimioterapia ou radioterapia de cabeça e pescoço ${ }^{(1)}$.

Desta forma, a amostra ficou constituída por 44 pacientes (88 orelhas), sendo 34 do gênero feminino $(77,3 \%)$ e 10 do gênero masculino $(22,7 \%)$.

Os pacientes selecionados foram encaminhados para o Setor de Fonoaudiologia da instituição para a realização da avaliação audiológica, composta por audiometria tonal liminar, audiometria vocal e imitância acústica, precedida da inspeção do meato acústico externo, para a investigação de anormalidades que pudessem alterar o resultado da avaliação.

$\mathrm{Na}$ audiometria tonal por via aérea, foram testadas as frequiências de $250 \mathrm{~Hz}$ a $8 \mathrm{kHz}$. Os tipos de perda auditiva foram classificados, de acordo com os critérios propostos por Silman e Silverman ${ }^{(20)}$. Quanto ao grau, as perdas auditivas foram classificadas de acordo com o critério proposto por Davis e Silverman ${ }^{(21)}$.

A imitância acústica foi realizada com tom de prova de $226 \mathrm{~Hz}$. Para classificar o tipo de timpanograma encontrado, foi utilizada a classificação de Jerger ${ }^{(22)}$.

Observou-se a presença ou ausência de reflexos estapedianos, considerando normais os que apareceram 70 $90 \mathrm{~dB}$ acima do limiar auditivo ${ }^{(23)}$.

A idade foi dividida nos seguintes intervalos de classes: 25 a 40 anos (adulto jovem), 41 a 59 anos (adulto) e 60 a 64 anos (idoso). Para o tempo de evolução da doença, utilizouse os intervalos inferior a dez anos e superior ou igual a dez anos, semelhantes aos empregados pelos autores pesqui$\operatorname{sados}^{(10-11)}$, permitindo a comparação com outros estudos.

Os dados foram apresentados sob a forma de tabelas e gráfico, sendo calculadas proporções. Para verificar a associação entre as variáveis, utilizou-se o Teste qui-quadrado $\left(c^{2}\right)$, considerando-se significância estatística pd"0,05.

\section{RESULTADOS}

A maioria dos sujeitos era do gênero feminino (77,3\%), evidenciando-se o predomínio da faixa etária de 41 - 59 anos $(61,4 \%)$ (Tabela 1). A média de idade foi de 53 anos.

Com relação ao tempo da doença, 31 pacientes $(70,4 \%)$ referiram diabetes há um tempo inferior a 10 anos; 8 pacientes $(18,2 \%)$ possuem o diagnóstico há um tempo superior ou igual a 10 anos. Cinco pacientes $(11,4 \%)$ não possuem referência quanto ao tempo do diagnóstico do diabetes (Tabela 2).

Vinte e oito pacientes $(63,6 \%)$ relataram hipertensão arterial, destes, $15(53,5 \%)$ apresentaram perda auditiva (Tabela 2).

Vinte e três pacientes $(52,2 \%)$ não apresentaram queixas auditivas, afirmando escutar normalmente. Dos pacientes que
Tabela 1. Distribuição quanto ao número (N) e porcentagem (\%) de pacientes diabéticos, segundo gênero e faixa etária (anos)

\begin{tabular}{|c|c|c|c|c|c|c|}
\hline \multirow{3}{*}{ Faixa etária } & \multicolumn{6}{|c|}{ Gênero } \\
\hline & \multicolumn{2}{|c|}{ Masculino } & \multicolumn{2}{|c|}{ Feminino } & \multicolumn{2}{|c|}{ Total } \\
\hline & $\mathrm{N}^{\circ}$ & $\%$ & $\mathrm{~N}^{\circ}$ & $\%$ & $\mathrm{~N}^{\circ}$ & $\%$ \\
\hline $25-40$ & 0 & 0 & 3 & 6,8 & 3 & 6,8 \\
\hline $41-59$ & 6 & 13,6 & 21 & 47,8 & 27 & 61,4 \\
\hline $60-64$ & 4 & 9,1 & 10 & 22,7 & 14 & 31,8 \\
\hline Total & 10 & 22,7 & 34 & 77,3 & 44 & 100 \\
\hline
\end{tabular}

Tabela 2. Distribuição dos pacientes diabéticos segundo sexo, presença de queixas, tempo de diagnóstico do diabetes, presença de hipertensão arterial e perda auditiva, além de comparação estatística

\begin{tabular}{|c|c|c|c|c|c|c|}
\hline \multicolumn{7}{|c|}{ Perda auditiva } \\
\hline & \multicolumn{2}{|c|}{ Sim } & \multicolumn{2}{|c|}{ Não } & \multirow[b]{2}{*}{ Teste } & \multirow[b]{2}{*}{$\mathrm{P}$} \\
\hline & $\mathrm{N}^{\circ}$ & $\%$ & $\mathrm{~N}^{\circ}$ & $\%$ & & \\
\hline \multicolumn{5}{|l|}{ 1. Sexo } & $0,78^{(1)}$ & 0,377 \\
\hline Masculino & 6 & 60,0 & 4 & 40,0 & & \\
\hline Feminino & 15 & 44,1 & 19 & 55,9 & & \\
\hline \multicolumn{5}{|l|}{ 2. Queixa } & $9,05^{(2)}$ & $0,003^{*}$ \\
\hline Sim & 15 & 71,4 & 6 & 28,6 & & \\
\hline Não & 6 & 26,1 & 17 & 73,9 & & \\
\hline \multicolumn{5}{|c|}{ 3. Tempo da doença } & $1,08^{(3)}$ & 0,298 \\
\hline$<10$ anos & 13 & 41,9 & 18 & 58,1 & & \\
\hline$\geq 10$ anos & 5 & 62,5 & 3 & 37,5 & & \\
\hline \multicolumn{5}{|c|}{ 4. Hipertensão arterial } & $1,05^{(4)}$ & 0,305 \\
\hline Sim & 15 & 53,6 & 13 & 46,4 & & \\
\hline Não & 6 & 37,5 & 10 & 62,5 & & \\
\hline
\end{tabular}

Legenda: N - Número; \% - Porcentagem; *(1) (2) (3) (4) c2

apresentaram queixas auditivas (21), $6(28,6 \%)$ referiram zumbido; 1 (4,7\%) hipersensibilidade a sons intensos; 6 $(28,6 \%)$ relataram apenas dificuldade auditiva; $4(19,0 \%)$ apresentaram dificuldade auditiva e zumbido; $1(4,7 \%)$ hipersensibilidade a sons intensos e zumbido; e $3(14,4 \%)$ apresentaram as três queixas anteriormente citadas. Ressalta-se que seis sujeitos, embora não mencionassem queixas, apresentaram perda auditiva (Tabela 2).

A perda auditiva encontrada nos pacientes avaliados foi do tipo neurossensorial, com predomínio em freqüências altas. Das 88 orelhas testadas, $33(37,5 \%)$ apresentaram perda auditiva, sendo 24 orelhas $(72,7 \%)$ de grau leve e 9 orelhas $(27,3 \%)$ de grau moderado. Verificou-se que 12 sujeitos $(27,3 \%)$ apresentaram perda auditiva bilateral (Tabela 3).

Tabela 3. Distribuição dos pacientes diabéticos, segundo resultado da avaliação auditiva

\begin{tabular}{lcc}
\hline Avaliação auditiva & $\mathrm{N}^{\circ}$ & $\%$ \\
\hline Normal & 23 & 52,3 \\
Perda unilateral & 9 & 20,4 \\
Perda bilateral & 12 & 27,3 \\
\hline Total & 44 & 100,0 \\
\hline
\end{tabular}

Legenda: $\mathrm{N}=$ número \% = porcentagem 
Com relação à faixa etária, nos pacientes de 25 - 40 anos, apenas uma orelha $(3,0 \%)$ apresentou perda auditiva; de 41 - 59 anos, 16 orelhas $(48,5 \%)$ apresentaram perda auditiva; e de 60 - 64 anos, 16 orelhas $(48,5 \%)$ apresentaram perda auditiva.

Analisando-se a perda auditiva em relação ao tempo da doença, verificou-se que 13 pacientes $(41,9 \%)$ com tempo de doença inferior a 10 anos apresentavam perda auditiva, enquanto que, no grupo com 10 ou mais anos de doença, 5 pacientes $(62,5 \%)$ eram portadores de déficit auditivo (Tabela 2).

Dos pacientes com diabetes por um tempo superior ou igual a 10 anos, 9 orelhas $(56,3 \%)$ apresentaram perda auditiva, sendo 4 na faixa etária de 41 - 59 anos e 5 de 60 - 65 anos. Os que tinham diabetes há menos de 10 anos apresentaram 19 orelhas $(30,6 \%)$ com perda auditiva, sendo um na faixa etária de 25 40 anos; 8 de 41 - 59 anos e 10 de 60 - 65 anos.

$\mathrm{Na}$ audiometria vocal, observou-se 86 orelhas $(97,7 \%)$ com índices de reconhecimento de fala (IRF) entre 92 e $100 \%$ para monossílabos; apenas duas orelhas $(2,3 \%)$ apresentaram IRF abaixo de $80 \%$ para monossílabos, sendo compatível com o grau da perda auditiva apresentado.

Em relação aos achados imitanciométricos, 71 orelhas $(80,7 \%)$ apresentaram curva timpanométrica do tipo A, 9 do tipo $\operatorname{Ar}(10,2 \%), 1$ do tipo Ad (1,2\%), 3 do tipo C $(3,4 \%)$ e 4 $(4,5 \%)$ não foram avaliadas, pois não foi possível o vedamento do meato acústico externo (Figura 1); constatou-se a presença de reflexo estapediano em 83 orelhas (94,3\%); ausência de reflexo em uma das orelhas que apresentava curva timpanométrica do tipo $\mathrm{C}$ e 4 orelhas não foram testadas.

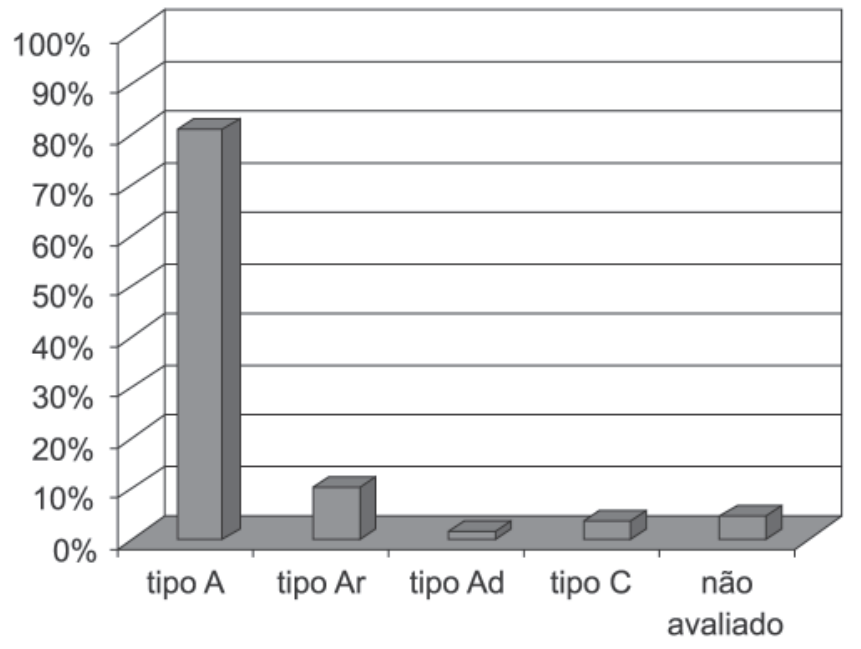

timpanograma

Figura 1. Distribuição dos pacientes diabéticos segundo resultado da timpanometria, analisando separadamente as orelhas

\section{DISCUSSÃO}

A maioria dos pacientes avaliados era do gênero feminino $(77,2 \%)$ e concentraram-se na faixa etária de 41 - 59 anos. Esses dados estão de acordo com as afirmações de que o diabetes do tipo II afeta principalmente adultos, especialmente após os 40 anos de idade, sendo mais comum em mulheres que homens ${ }^{(6,24)}$.

Com relação ao tempo de evolução do diabetes, embora a proporção de perda auditiva entre os pacientes com tempo de evolução maior ou igual a dez anos seja mais elevada que entre os pacientes com diabetes há menos de dez anos, não houve associação estatisticamente significante entre tempo da doença e perda auditiva $\left(\chi^{2}=1,08 ; p=0,298\right)$. Em estudo realizado por outros autores, das 72 orelhas testadas de pacientes diabéticos, $44(61,1 \%)$ apresentaram alterações ${ }^{(10)}$.

A hipertensão arterial foi referida em $63,6 \%$ dos pacientes avaliados, desses, 53,5\% apresentaram perda auditiva. No entanto, a associação não foi estatisticamente significante $\left(\chi^{2}=1,05 ; \mathrm{p}=0,305\right)$. Alguns autores estudaram a relação entre hipertensão arterial e perda auditiva neurossensorial, sugerindo a hipótese de que a hipertensão arterial lesa o órgão sensorial da cóclea, o que seria responsável por uma perda auditiva neurossensorial. Este seria um fator a considerar na aparição e progressão da perda auditiva em pessoas com diabetes tipo $\mathrm{II}^{(14)}$.

Zumbido e dificuldade para escutar foram queixas auditivas mais freqüentemente referidas pelos pacientes. Estudos utilizados como referência relataram que 72,2\% dos pacientes diabéticos referiram zumbido como queixa auditiva, contra 58,3\% do grupo controle. Alguns autores colocaram que pode haver a existência de zumbido em problemas metabólicos ${ }^{(10)}$. Verificou-se uma associação estatisticamente significante entre queixas e perda auditiva $\left(\chi^{2}=9,05 ; \mathrm{p}=\right.$ $0,003)$, ressaltando a importância de proceder a avaliação auditiva precoce nos pacientes com essas queixas.

Em todos os pacientes em que foi observada perda auditiva, a mesma foi do tipo neurossensorial, com predomínio nas frequiências altas, de grau leve e moderado, porém com uma maior porcentagem de perdas de grau leve. Esse resultado está de acordo com vários estudos realizados por diversos autores $^{(10-11,16)}$, cujos resultados revelaram perda auditiva em todos os casos, do tipo neurossensorial, independente do tempo de evolução do diabetes, havendo um predomínio de graus leve e moderado e afetando mais as freqüências altas ${ }^{(11)}$.

Com relação à faixa etária, observou-se que a ocorrência de perda auditiva foi mais expressiva na faixa etária de 60 a 65 anos $(71,4 \%)$, seguida da faixa de 41 a 59 anos $(37,0 \%)$, representado 33,3\% entre os de 25 a 40 anos. Estudo semelhante também revelou um considerável aumento do número de perdas auditivas, com o acréscimo da idade, afirmando não encontrar limiares auditivos dentro dos padrões de normalidade nos grupos de diabéticos com idades superiores a 61 anos $^{(10)}$.

Esses resultados podem estar sendo influenciados pela presença de presbiacusia. Chama-se a atenção para a dificuldade em se distinguir a perda auditiva dos diabéticos da perda auditiva senil, em indivíduos não-diabéticos. Alguns estudos indicam que o decréscimo na acuidade auditiva, que é similar ao apresentado na presbiacusia, é maior do que o esperado pela idade, em pacientes mais idosos e diabéticos ${ }^{(13)}$. É mais provável uma associação de fatores que levariam a uma perda auditiva, como conseqüência da alteração vascular provocada pelo diabetes ${ }^{(3)}$. 
Não foram encontrados estudos específicos que mostrassem dados relacionados aos testes de reconhecimento de fala em pacientes diabéticos. Alguns autores referem apenas que alterações nas células cerebrais em alguns diabéticos podem resultar em processamento mais lento de sons complexos como os da fala ${ }^{(2)}$. Nossos resultados mostraram IRF compatível com os limiares tonais encontrados.

Inúmeros estudos clínicos têm investigado a possível associação entre diabetes e perda auditiva; porém, muitos desses estudos avaliaram entre 20 e 50 pacientes, o que pode limitar a habilidade de detectar qualquer associação. Autores referiram discreta associação entre diabetes do tipo II e perda auditiva, após avaliarem 3.373 indivíduos, sendo 344 portadores de diabetes ${ }^{(19)}$. Entretanto, nosso estudo foi desenvolvido com uma amostra pequena de pacientes, pelo fato de se ter proposto a avaliar somente pacientes diabéticos atendidos no Setor de Diabetes do NAMI.

Muitas questões poderão ser esclarecidas por meio de estudos longitudinais, multicêntricos, com grande número de pacientes e critérios rígidos de inclusão e exclusão ${ }^{(3)}$.

\section{Limitações do estudo}

Os pacientes forneceram informações muitas vezes imprecisas ou não sabiam informar sobre dados importantes, dificultando a coleta de dados. Questões sobre presença ou ausência de hipertensão arterial ou tempo de evolução da doença, por exemplo, não puderam ser checadas, aceitando como verdade o que foi referido pelo paciente. Acredita-se que distúrbios vasculares associados à hipertensão arterial sistêmica possam ser etiologicamente responsáveis pela perda auditiva ${ }^{(19)}$. Por outro lado, outros estudos não encontraram relação entre a presença de hipertensão arterial sistêmica e diabetes mellitus e a piora nos limiares audiométricos ${ }^{(18)}$.

Não foram realizados exames complementares relacionados ao diabetes, como a taxa de glicemia. Também, não foi investigada presença de neuropatia diabética periférica, nefropatia diabética e retinopatia. Pesquisadores referiram que existe piora dos limiares em frequiências altas, proporcionalmente ao tempo de evolução da doença e ao descontrole da glicemia ${ }^{(18)}$. Pesquisas encontraram limiares audiométricos acentuadamente piores em diabéticos com neuropatia diabé- tica, quando comparados à população controle e aos diabéti$\cos$ não-neuropatas ${ }^{(25)}$.

\section{CONCLUSÃO}

A análise dos resultados evidencia que os participantes do estudo eram, na sua maioria, do gênero feminino, encontravam-se na faixa etária de 41 a 59 anos e eram portadores da doença há menos de 10 anos.

Uma elevada proporção referiu queixas auditivas, sendo mais freqüente o zumbido; porém, vários sujeitos apresentaram a associação de várias queixas. Quanto à perda auditiva, esta se encontrava presente em quase metade dos diabéticos, sendo do tipo neurossensorial, de grau leve e moderado, com predomínio em freqüências altas.

Após a execução deste estudo, podemos observar que a audição pode estar comprometida em pacientes com diabetes do tipo II. Porém, os fatores causais ainda não estão bem definidos e vários estudos estão sendo desenvolvidos para tentar elucidar a provável etiopatogenia do diabetes. A fisiopatologia da alteração ainda é muito discutida pelos diversos autores.

O desenvolvimento de pesquisas também seria importante para auxiliar no diagnóstico precoce do diabetes, o que é de extrema relevância, já que evitaria o desenvolvimento das complicações decorrentes da doença, inclusive a prevenção das possíveis perdas auditivas.

Destaca-se a importância da identificação precoce de perdas auditivas em pacientes diabéticos e do processo de reabilitação, por meio de próteses auditivas, evitando, assim, que a qualidade de vida desses pacientes seja prejudicada pelos transtornos que esta alteração acarreta.

Seria fundamental a criação de programas de triagem, que pudessem identificar prováveis portadores de diabetes, pois, a grande maioria dos pacientes apresenta diabetes de forma assintomática e, devido a isso, recebem diagnóstico tardio, aumentando assim as chances de complicações crônicas.

Baseado nos dados obtidos neste estudo, recomenda-se a avaliação audiológica de pacientes portadores de diabetes do tipo II, fazendo parte da rotina clínica, buscando a identificação precoce de possíveis alterações auditivas que podem afetar a qualidade de vida destes pacientes.

\begin{abstract}
Purpose: People that suffer from Diabetes Mellitus (DM) type II constitute a risk population for hearing disorders, since metabolic disease can affect nerve and vascular tissues that attain directly the auditory system. The present study investigated the audiologic profile of patients with DM type II. Methods: A descriptive study was carried out, where 44 patients (88 ears) were evaluated. The ages of the subjects ranged from 25 to 65 years. All subjects underwent an audiological evaluation that included pure tone audiometry, speech audiometry and acoustic immittance measures. Results: The prevalence of patients with DM type II was higher among female $(77.3 \%)$. Thirty one (70.4\%) patients had DM type II for less than 10 years. Thirty three ears (37.5\%) had sensorineural hearing loss ranging from low to moderate degree, predominantly in high frequencies. In the acoustic immittance measures, 71 (80,7\%) ears showed type A tympanograms and 83 (94,3\%) ears had presence of acoustic reflex. Conclusion: The occurrence of hearing loss was observed in patients with DM type II; consequently, the auditory evaluation of these patients is important to the follow up of the hearing loss.
\end{abstract}

Keywords: Diabetes mellitus, type 2/diagnosis; Diabetes mellitus, type 2/complications; Blood glucose; Hearing loss/etiology 


\section{REFERÊNCIAS}

1. Salvinelli F, Miele A, Casale M, Greco F, D'Ascanio L, Firrisi L, et al. Hearing thresholds in patients with diabetes. Internet J Otorhinolaryngol [serial on the Internet]. 2004 [cited 2007 Nov 12]; 3(1):[about 8 p.]. Available from: http://www.ispub.com/ostia/index.php?xmlFilePath= journals/ijorl/vol3n1/diabetes.xml

2. Vaughan N, McDermott D. Diabetes and hearing loss: exploring connections. Hear Health [serial on the Internet]. 2003 [cited 2007 Noy 12]; 19(3). Available from: em: http://www.drf.org/hearing_health/ archive/2003/fall03_diabetesandhearingloss.htm

3. Maia CAS, Campos CAH. Diabetes Mellitus como causa de perda auditiva. Rev Bras Otorrinolaringol. 2005;71(2):208-14.

4. Almeida CA, Vieira Neto L, Vaisman M. Como tratar e diagnosticar diabetes. RBM Rev Bras Med. 2004;61:12-20.

5. Macedo CLD, Balestro AC, Ribas FE, Faccini G, Bringhenti RL. Diabetes mellitus e sua relação com outras co-morbidades [texto na Internet]. 2000. [citado 2005 Dez 02]. Disponível em: http:// www.saudebrasilnet.com.br/premios/saude/premio2/trabalhos/037.pdf

6. Guardabassi A. Diabetes: mitos e verdades. In: Vox Scientiae [serial on the Internet]. 2004 [citado 2007 Nov 12]; 4(21) Disponível em: http:// www.eca.usp.br/nucleos/njr/voxscientiae/andreA21.html.

7.Silva FML, Silva MMML. Diabetes mellitus do tipo II nos dias atuais. J Bras Med. 2005;88(1/2):13-22.

8. Melo KFS, Giannella MLCC, Fidelix MSP, Nery M, Giannella Neto D. Diabetes mellitus. RBM Rev Bras Med. 2003;60(7):505:509:514: passim-508-512-516.

9. Dall'Igna C, Batista LRP, Siqueira MK. Patogênese da disacusia neurossensorial em diabetes mellitus. Rev Bras Otorrinolaringol. 2000;66(2):155-8

10. Marchiori LLM, Gibrin PCD. Diabetes mellitus: prevalência de alterações auditivas. Arq Bras Endocrinol Metab. 2003;47(1):82-6.

11. Perera Delgado JI, Licea Puig ME. Afectación de la audición en personas con diabetes mellitus tipo 2. Av Diabetol. 2003;19(3):123-9.

12. Bezerra B. Diabetes e hipertensão: cuidado duplo [texto na internet]. 2006. [citado 2007 Jul 7]. Disponível em: http://www.diabetes.org.br/ imprensa/entrevistas/index.php?id=266
13. Fuess VLR, Cerchiari DP. Estudo da hipertensão arterial sistêmica e do diabetes mellitus como fatores agravantes da presbiacusia. Arq Otorrinolaringol. 2003;7(2):116-21.

14. Duck SW, Prazma J, Bennett PS, Pillsbury HC. Interaction between hypertension and diabetes mellitus in the pathogenesis of sensorineural hearing loss. Laryngoscope. 1997;107(12 Pt 1):1596-605.

15. Davanipour Z, Lu NM, Lichtenstein M, Markides KS. Hearing problems in Mexican American elderly. Am J Otol. 2000;21(2):168-72.

16. Bittar RSM, Sanchez TG, Santoro PP, Medeiros IRT. O metabolismo da glicose e o ouvido interno. Arq Otorrinolaringol. 1998;2(1):4-8.

17. Hopkins M. Diabetes may be linked to early hearing loss. Nature News [periódico na Internet]. 2004. [citado 2005 Abr 10]. Available from: http://www.nature.com/news/2004/040223/full/news040223-12.html.

18. Elliot VS. Possible correlation between diabetes and hearing loss. AMNews [text on the Internet]. 2004; 15. [cited 2005 Mai 25]. Available from: http://www.ama-assn.org/amednews/2004/03/15/hlsc0315.htm

19. Dalton DS, Cruickshanks KJ, Klein R, Klein BE, Wiley TL. Association of NIDDM and hearing loss. Diabetes Care. 1998;21(9):1540-4. Comment in: Diabetes Care. 1999;22(1):180.

20. Silman S, Silverman CA. Auditory diagnosis: principles and applications. San Diego: Singular Publishing Group; 1997. p.48-62.

21. Davis H, Silverman SR. Hearing and deafness. New York: Holt, Rinehart and Winston; 1970.

22. Katz J. Tratado de audiologia clínica. 4a ed. São Paulo: Manole; 1999. p. 281-97.

23. Lopes Filho OC. Contribuição ao estudo clínico da impedância acústica [tese]. São Paulo: Faculdade de Medicina da Universidade de São Paulo; 1972.

24. Chartrand MS. Diabetes \& Hearing Health 2003. Hearing Health [homepage on the Internet], 2003. [cited 2005 Dez 2]. HIPAA; c2007.Available from: http://www.digicare.org/diabetes-hearing.asp. [Reprinted from Audiology Online July 30, 2003]

25. Acuña Garcia M, Herrero Laso JL, Durán Díez C, Menéndez Argüelles ME, Vallejo Valdezate LA, Díaz Suárez I, et al. Complicaciones diabéticas e hipoacusia. An Otorrinolaringol Ibero Am. 1997;24(5):465-76. 